ARTICLE

DOI: $10.1038 / \mathrm{s} 41467-017-00516-5$

OPEN

\title{
Strain-engineered growth of two-dimensional materials
}

Geun Ho Ahn'1,2, Matin Amani 1,2, Haider Rasool 2,3,4, Der-Hsien Lien (D) 1,2, James P. Mastandrea ${ }^{2,3}$, Joel W. Ager III (10) 2,3, Madan Dubey ${ }^{5}$, Daryl C. Chrzan ${ }^{2,3}$, Andrew M. Minor 2,3,4 \& Ali Javey (1) 1,2

The application of strain to semiconductors allows for controlled modification of their band structure. This principle is employed for the manufacturing of devices ranging from highperformance transistors to solid-state lasers. Traditionally, strain is typically achieved via growth on lattice-mismatched substrates. For two-dimensional (2D) semiconductors, this is not feasible as they typically do not interact epitaxially with the substrate. Here, we demonstrate controlled strain engineering of $2 \mathrm{D}$ semiconductors during synthesis by utilizing the thermal coefficient of expansion mismatch between the substrate and semiconductor. Using $\mathrm{WSe}_{2}$ as a model system, we demonstrate stable built-in strains ranging from $1 \%$ tensile to $0.2 \%$ compressive on substrates with different thermal coefficient of expansion. Consequently, we observe a dramatic modulation of the band structure, manifested by a strain-driven indirect-to-direct bandgap transition and brightening of the dark exciton in bilayer and monolayer $\mathrm{WSe}_{2}$, respectively. The growth method developed here should enable flexibility in design of more sophisticated devices based on 2D materials.

\footnotetext{
${ }^{1}$ Electrical Engineering and Computer Sciences, University of California at Berkeley, Berkeley, CA 94720, USA. ${ }^{2}$ Materials Sciences Division, Lawrence Berkeley National Laboratory, Berkeley, CA 94720, USA. ${ }^{3}$ Department of Materials Science, University of California at Berkeley, Berkeley, CA 94720, USA. ${ }^{4}$ National Center for Electron Microscopy, Molecular Foundry, Lawrence Berkeley National Laboratory, Berkeley, CA 94720, USA. ${ }^{5}$ US Army Research Laboratory, 2800 Powder Mill Road, Adelphi, MD 20783, USA. Geun Ho Ahn and Matin Amani contributed equally to this work. Correspondence and requests for materials should be addressed to A.J. (email: ajavey@eecs.berkeley.edu)
} 
T wo-dimensional (2D) transition metal dichalcogenides (TMDCs) have been the subject of focused research owing to their potential applications in optoelectronics and sub $10 \mathrm{~nm}$ transistors ${ }^{1,2}$. The primary attraction of TMDCs such as $\mathrm{MoS}_{2}$ and $\mathrm{WSe}_{2}$ for both applications is their naturally terminated surface, which allows them to be scaled down to the atomic limit without the concern of surface dangling bonds. Furthermore, in many $2 \mathrm{D}$ materials, a number of desirable properties emerge at the monolayer limit, the most notable of which being the presence of a direct bandgap. Many studies based on mechanical bending of exfoliated 2D TMDCs have been conducted on flexible substrates, and they have shown that the application of strain can tune the properties of this new class of materials ${ }^{3-6}$. For example, it has been demonstrated that in multilayer $\mathrm{WSe}_{2}$, particularly in nominally indirect-gap bilayer $\mathrm{WSe}_{2}$, application of tensile strain can result in a transition from an indirect-to-direct bandgap ${ }^{7}$. However, studies on the effects of strain in TMDCs have been limited to mechanical bending of samples, and there has yet to be a technique, which can directly realize built-in tensile and compressive strains.

Traditionally, strain engineering of semiconductors has been leveraged to tune the electronic band structure of high-performance devices, the most notable of which being to reduce intervalley scattering, increase mobility in Si transistors, and reduce the hole effective mass in III-V semiconductor lasers ${ }^{8}$, 9 . Growth on epitaxial substrates with a controlled lattice constant mismatch has typically been utilized to establish built-in strain in three-dimensional semiconductors ${ }^{10}$. However, due to the relatively weak interaction between $2 \mathrm{D}$ materials and substrates, this established method of strain engineering is likely not applicable for the strain-engineered growth of TMDCs. Therefore, a route toward development of large-area strained TMDCs on a practical substrate is highly desirable. Significant research efforts have been made to realize large area TMDCs, with the majority of efforts focusing on chemical vapor deposition (CVD ${ }^{11-13}$. Interestingly, a number of studies employing CVD growth of 2D materials have reported apparent strain in the synthesized samples ${ }^{14}, 15$.
However, growth of high-quality 2D materials with controllable built-in strain has not been realized. Hence, properties that are difficult to probe via mechanical bending experiments, such as low-temperature optical measurements and electrical performance, have yet to be explored.

In this work, we demonstrate strain engineering of $2 \mathrm{D}$ materials directly via CVD growth while simultaneously maintaining high material quality, by utilizing the thermal coefficient of expansion (TCE) mismatch between the TMDC and the growth substrate. Electron diffraction of strained monolayers grown directly onto transmission electron microscopy (TEM) windows is utilized to unambiguously quantify strain. Using $\mathrm{WSe}_{2}$ as a model system, we show that it is possible to obtain both tensile (of $\sim 1 \%)$ and compressive $(0.2 \%)$ strained $2 \mathrm{D}$ semiconductors over large areas on rigid substrates. Furthermore, in the $\mathrm{WSe}_{2}$ model system we show indirect-to-direct optical transition in tensile strained $\mathrm{WSe}_{2}$ bilayers as well as removal of dark exciton quenching in $\mathrm{WSe}_{2}$ monolayers.

\section{Results}

TCE-mismatch induced strain. Owing to the high growth temperatures used to synthesize TMDCs, the TCE mismatch between the substrate and the $2 \mathrm{D}$ semiconductor can be utilized to control the strain in the synthesized 2D material, as shown schematically in Fig. 1. The theoretical upper limit for the strain, assuming no relaxation, can be calculated using the difference in lattice constant of the substrate and 2D semiconductor at room temperature and synthesis temperature:

$$
\varepsilon\left(T_{\mathrm{g}}\right)=\frac{a_{2 \mathrm{D}}\left(T_{\mathrm{g}}\right)-a_{2 \mathrm{D}}\left(25^{\circ} \mathrm{C}\right)}{a_{2 \mathrm{D}}\left(25^{\circ} \mathrm{C}\right)}-\frac{a_{\mathrm{Sub}}\left(T_{\mathrm{g}}\right)-a_{\mathrm{Sub}}\left(25^{\circ} \mathrm{C}\right)}{a_{\mathrm{Sub}}\left(25^{\circ} \mathrm{C}\right)}
$$

where $a_{2 \mathrm{D}}$ is the in-plane lattice constant for the $2 \mathrm{D}$ material being grown, $a_{\text {Sub }}$ is the in-plane lattice constant of the substrate, and $T_{\mathrm{g}}$ is the growth temperature. More generally, this can be calculated using the temperature dependent thermal expansion coefficient for the $2 \mathrm{D}$ material $\left(\alpha_{2 \mathrm{D}}\right)$ and substrate $\left(\alpha_{\mathrm{Sub}}\right)$

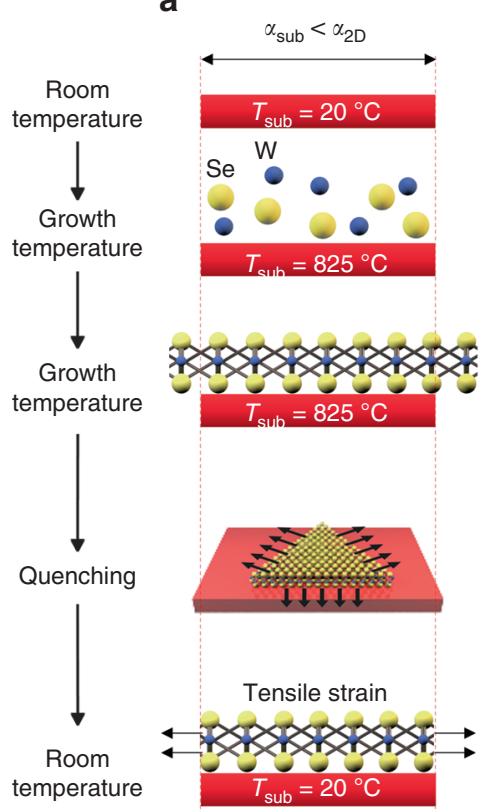

b

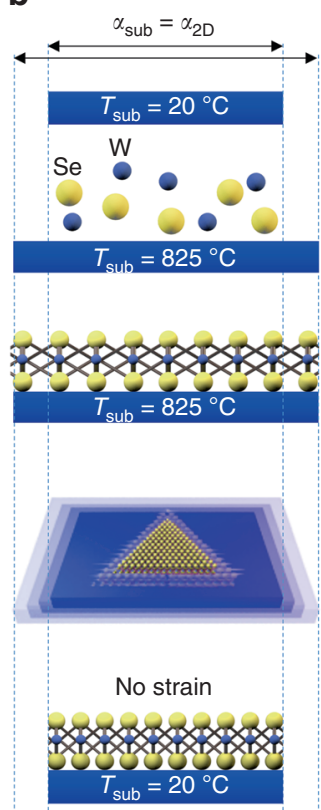

c

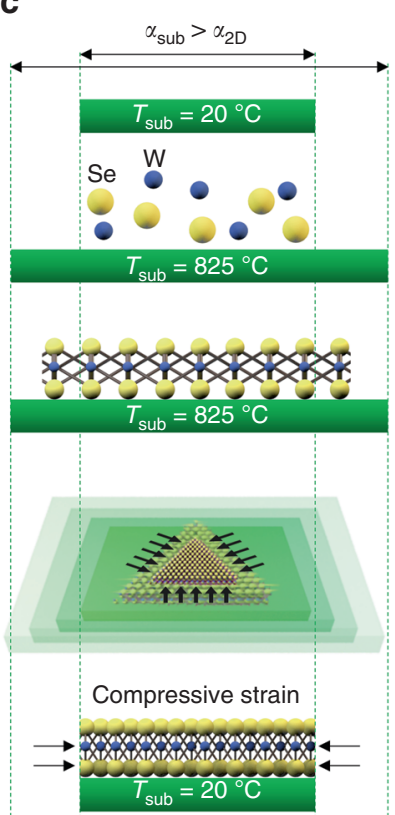

Fig. 1 Strain-engineered growth of WSe $e_{2}$ using TCE-mismatch. Schematic of the process through which strain is realized during the growth of 2D materials via mismatch in substrate and TMDC thermal expansion coefficient. a Tensile strain is achieved when the TCE of the substrate is less than that of the 2D material, $\mathbf{b}$ relaxed samples are achieved when the TCE of the substrate and 2D material match, and $\mathbf{c}$ compressive strain is achieved when the TCE of the substrate is greater than that of the $2 \mathrm{D}$ material 
a

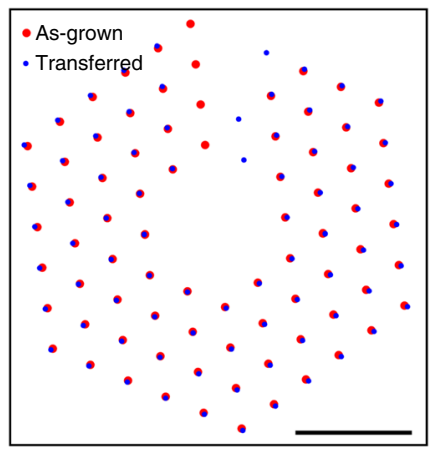

d

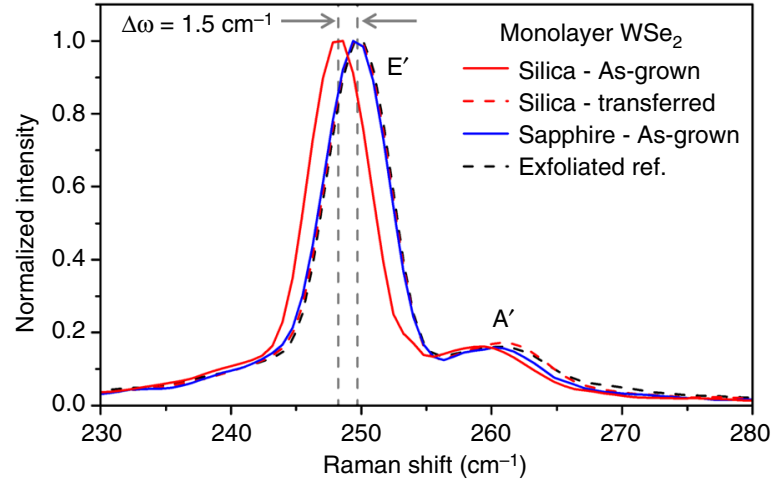

b

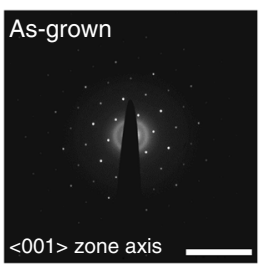

C

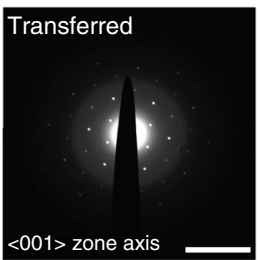

e

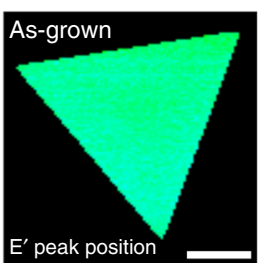

$\mathbf{f}$

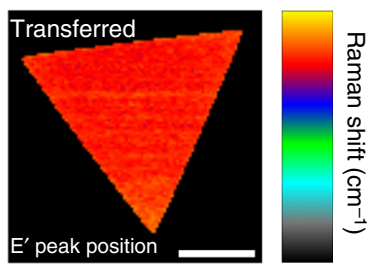

Fig. 2 Transmission electron microscopy images and Raman spectroscopy of monolayer $\mathrm{WSe}_{2}$. a Overlay of diffraction spots for as-grown and transferred monolayer $\mathrm{WSe}_{2}$, showing a $1.39 \pm 0.28 \%$ strain in samples grown directly on the TEM membrane. Scale bar is $10 \mathrm{~nm}^{-1}$. $\mathbf{b}$ Electron diffraction patterns of a

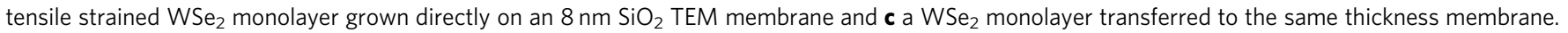
Scale bar is $10 \mathrm{~nm}^{-1}$. d Raman spectra of $\mathrm{WSe}_{2}$ monolayers grown on various substrates. e, $\mathbf{f}$ Raman mapping of $\mathrm{E}^{\prime}$ peak position for a (e) tensile strained $\mathrm{WSe}_{2}$ monolayer as-grown on fused silica and a (f) $\mathrm{WSe}_{2}$ monolayer transferred to release strain; data are plotted using the same false color scheme. Scale bar is $20 \mu \mathrm{m}$, data range is 250.5 to $247.0 \mathrm{~cm}^{-1}$

according to:

$$
\varepsilon\left(T_{\mathrm{g}}\right)=\int_{25^{\circ} \mathrm{C}}^{T_{\mathrm{g}}} \alpha_{2 \mathrm{D}}(T) \mathrm{d} T-\int_{25^{\circ} \mathrm{C}}^{T_{\mathrm{g}}} \alpha_{\text {Sub }}(T) \mathrm{d} T
$$

To retain the strain generated from TCE-mismatch, the bonding between the $2 \mathrm{D}$ layer and the substrate must be sufficiently strong and corrugated to maintain the nonslip condition that transmits strain to the layer. Although one expects strains of this magnitude to arise during epitaxial growth, it is difficult to see how a similar strain can arise for films bound by van der Waals forces to an amorphous substrate. Experimentally, the corrugation in the film must be large enough to retain the strain upon cooling. To estimate the magnitude of the corrugation necessary to sustain the strain, we analyzed a 2D Frenkel-Kontorova model ${ }^{16}$, shown in Supplementary Note 1 and Supplementary Fig. 1. This model suggests that atomic binding energy variations of as little as $14 \mathrm{meV}$ over the range of the unit cell are capable of retaining strains of the magnitude of those observed experimentally. This variation is $\sim 1 \%$ of a typical covalent bond strength, and very near to the typical strength of a van der Waals bond. Though a more detailed theory needs to be developed, especially to understand the surface chemistry immediately prior to growth to determine the nature of the bonding, this simple analysis suggests that thermal-expansion mismatch can strain a film, even in the case of weak binding between the film and the substrate. It should be noted that in a previous study, local heating of $\mathrm{MoS}_{2}$ using a laser was used to generate strains of up to $0.2 \%$ arising from the TCE-mismatch of $\mathrm{MoS}_{2}$ with the substrate ${ }^{17}$. Although this method does not generate built-in strains (strain is released once the laser is turned off), it suggests that the $\mathrm{MoS}_{2}$-substrate bonding is strong enough to enable TCE-mismatched induced strain engineering ${ }^{17}$.

To demonstrate strain-engineered CVD growth of $\mathrm{WSe}_{2}$, four substrates were chosen, which have a range of TCEs. Fused silica and aluminum nitride (AlN) have a TCE of 0.55 and $5.5 \mathrm{ppm}$, respectively, much smaller than that of $\mathrm{WSe}_{2}(9.5 \pm 3.2 \mathrm{ppm})$, and would be expected to induce tensile strain $^{3}, 18-\overline{2} 2$. In contrast, sapphire has a TCE closely matched to $\mathrm{WSe}_{2}$ and would be expected to produce relaxed samples and strontium titanate (STO) with a TCE of 12 ppm should yield compressively strained samples $20,23,24$. The sample was rapidly quenched after growth to limit relaxation in the $\mathrm{WSe}_{2}$. It is important to note that the rapid quenching process was found to be important in maintaining the presence of strain, and delay of the quenching step can result in partial relaxation of the $2 \mathrm{D}$ material.

Characterization strain and material quality. To characterize the strain present in the as-grown material, multiple characterization methods were utilized. First, electron diffraction was performed on $\mathrm{WSe}_{2}$ as-grown on $\mathrm{SiO}_{2}$ TEM grids and $\mathrm{WSe}_{2}$ transferred to $\mathrm{SiO}_{2}$ TEM grids. The resulting diffraction patterns are shown in Figs. 2a-c. In all TEM diffraction measurements on $\mathrm{WSe}_{2}$, the camera length and lens aberrations of the imaging systems were calibrated using polycrystalline aluminum and single crystal aluminum calibration samples (Ted Pella), and the microscope lens settings were left constant for all subsequent measurements. Corresponding images and photoluminescence (PL) spectra of the samples in Figs. 2b, c are shown in Supplementary Fig. 2. By comparing the lattice constant extracted from the diffraction patterns of strained (as-grown) and unstrained (transferred) $\mathrm{WSe}_{2}$, and utilizing the center of mass for 75 diffraction spots from each pattern, we calculate that a 1.39 $\pm 0.28 \%$ tensile strain is present in the as-grown sample. The 
calculated error represents the standard deviation in lattice constant value over 75 measured diffraction spots. Raman spectrum shown in Fig. 2d reveals a shift of the E' in-plane Raman mode of $1.5 \pm 0.2 \mathrm{~cm}^{-1}$ between as-grown and transferred $\mathrm{WSe}_{2}$. We note that the strain measured here is biaxial, not uniaxial, in contrast to mechanical bending studies and thus we do not observe splitting of the $E^{\prime}$ mode. Therefore, the theoretically calculated value for the Grüneisen parameter $(\chi)$ of $\mathrm{WSe}_{2}$ is used to calculate the expected peak shift according to: $\omega(\varepsilon)=\omega_{0}+\chi \varepsilon$, which is consistent with the approximately $1 \%$ tensile strain measured via diffraction $^{25-27}$. It should also be noted that after transfer the E' mode shows no shift relative to samples prepared either by exfoliation or as-grown on sapphire. High resolution Raman mapping was performed and is shown in Figs. 2e, $\mathrm{f}$ for $\mathrm{WSe}_{2}$ crystals as-grown on fused silica and transferred to a new fused silica substrate, respectively. We observe a uniform Raman shift throughout the full $\mathrm{WS}_{2}$ domain for both samples indicating that the strain is uniformly distributed within the sample on the scale of the Raman spot size. In addition, PL and atomic force microscopy images of as-grown $\mathrm{WSe}_{2}$ on fused silica are shown in Supplementary Fig. 3.

PL measurements were employed to measure the bandgap as a function of strain. Figure 3a shows PL spectra of samples grown on all substrates investigated in this work as well as an exfoliated reference. There is a large spectral shift of $120 \mathrm{meV}$ between samples grown on fused silica with near zero TCE and STO, which has the highest TCE of all substrates investigated in this work. Figure $3 \mathrm{~b}$ shows the estimated strain from the PL peak shift as a function of predicted strain in $\mathrm{WSe}_{2}$, vs. the lattice mismatch between the substrate and TMDC lattice constant. Strain values are determined from the experimentally measured PL peak position (measurement error was calculated using the standard deviation of strain measured from fifteen different crystals) and theoretically calculated bandgap as a function of strain extracted from refs 3,27 . It is important to note that we utilized the difference in peak position relative to an unstrained sample to eliminate effects from absolute bandgap error relative to theoretical calculations. We performed an analysis of the expected strain due to lattice parameter misfit (Supplementary Table 1 and Supplementary Note 2) to rule out the influence of epitaxial strain between the substrate and $\mathrm{WSe}_{2}$. From this calculation, the expected strain from the lattice mismatch shows the opposite trend as compared with what we observe experimentally. We find that our experimentally realized strain per $\Delta \mathrm{TCE}$ of $-0.10 \pm$ $0.01 \%$ per $\mathrm{ppm} \mathrm{K}^{-1}$ is in excellent agreement with the theoretical value of $-0.09 \%$ per $\mathrm{ppm} \mathrm{K}^{-1}$. To verify that the substrate does not affect the PL peak shape or position of monolayer $\mathrm{WSe}_{2}$, samples were transferred to the four substrates used in this study (Supplementary Fig. 4) and no shift of the PL peak was observed. The impact of growth temperature to tune the strain was also studied using fused silica as the substrate. Figure $3 c$ shows the tensile strain in monolayer $\mathrm{WSe}_{2}$ grown at different temperatures, estimated using PL peak position. We are able to tune the strain from $0.94 \pm 0.06 \%$ to $0.67 \pm 0.05 \%$ as the growth temperature is changed from 900 to $673^{\circ} \mathrm{C}$. PL spectra corresponding to Fig. $3 \mathrm{c}$ is shown in Supplementary Fig. 5.

Tensile strain engineering of mono- and bilayer $\mathrm{WSe}_{2}$. Calibrated PL measurements were used to provide a gauge of material quality. We find that strained $\mathrm{WSe}_{2}$ monolayers as-grown on fused silica show comparable PL intensity to exfoliated $\mathrm{WSe}_{2}$, demonstrating that strain engineering of $2 \mathrm{D}$ materials can be achieved without compromising material performance. Specifically, as exfoliated samples show a typical quantum yield (QY) of $2.3 \pm 0.4 \%$, while samples grown on fused silica show a typical QY of $1.9 \pm 0.6 \%$. Upon transfer, the strain within the as-grown
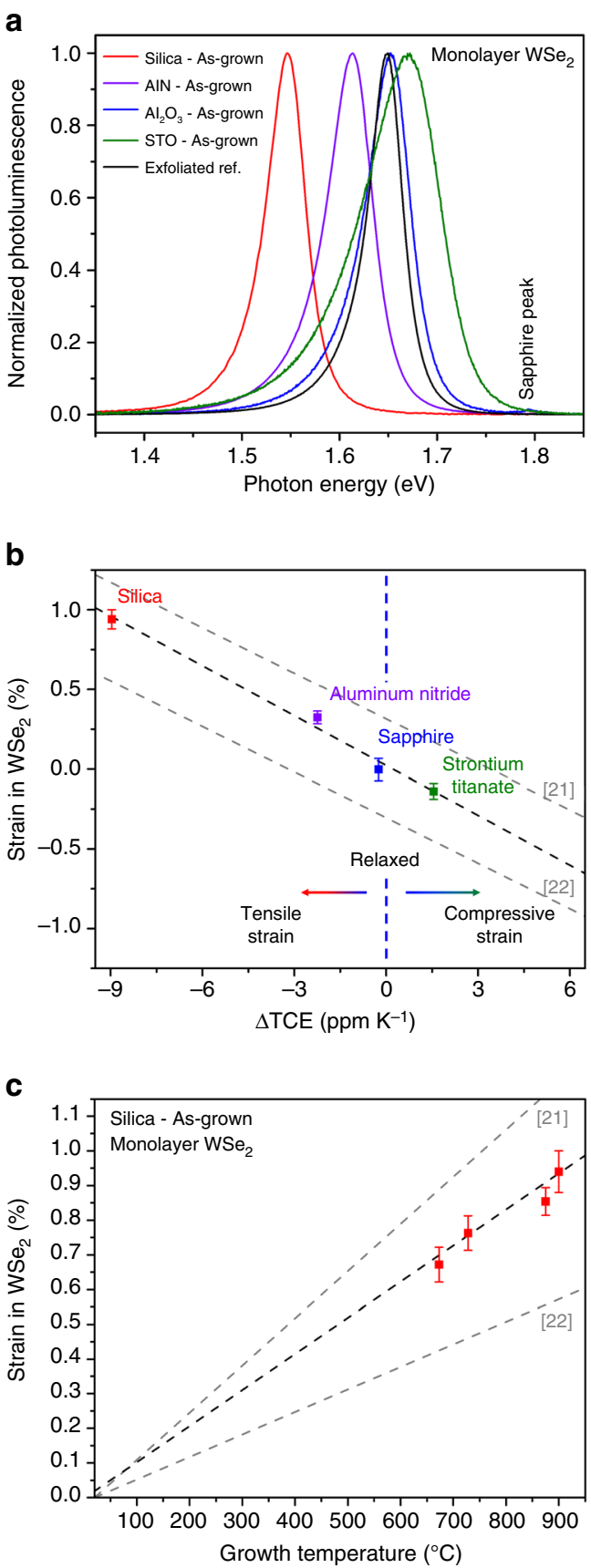

Fig. 3 Strain engineering of $2 \mathrm{D}$ materials. a PL spectra for monolayer $\mathrm{WSe}_{2}$ as-grown on substrates with a range of thermal coefficient of expansion mismatches. $\mathbf{b}$ Estimated strain that can be achieved in 2D materials based on the mismatch between the substrate and TMDC thermal coefficient of expansion; data points show measured strain for substrates with a range of thermal expansion coefficient mismatch; dashed gray lines are based on TCE values for $\mathrm{WSe}_{2}$ obtained from refs 21,22 , dashed black line shows fit to experimental data. c Estimated strain realized in $\mathrm{WSe}_{2}$ as-grown on fused silica at various substrate temperatures; dashed gray lines are based on TCE values for $\mathrm{WSe}_{2}$ obtained from refs ${ }^{21,22}$, dashed black line shows fit to experimental data. Error bars indicate standard deviation of strain, measured over fifteen samples

material is released and its emission peak shifts to $1.65 \mathrm{eV}$, closely matching that of exfoliated samples, as shown in Fig. 4a. In addition to emission, the absorption spectrum of the as-grown monolayer was measured and is shown in Fig. 4b. The absorption spectra clearly shows that the A and B exciton resonances are red- 
a

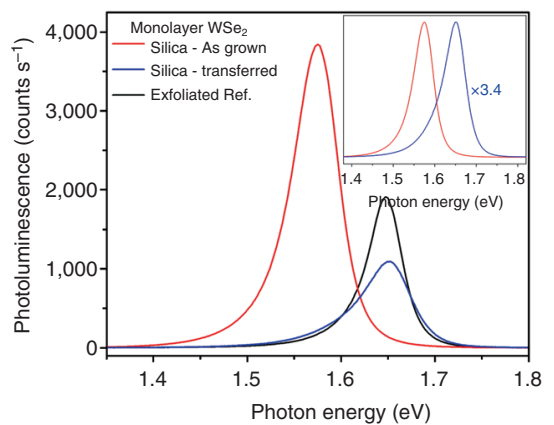

C

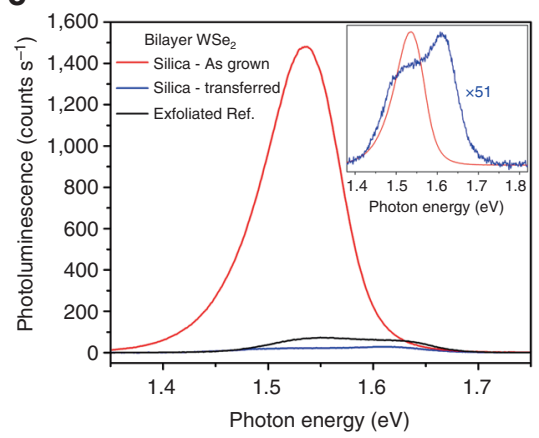

b

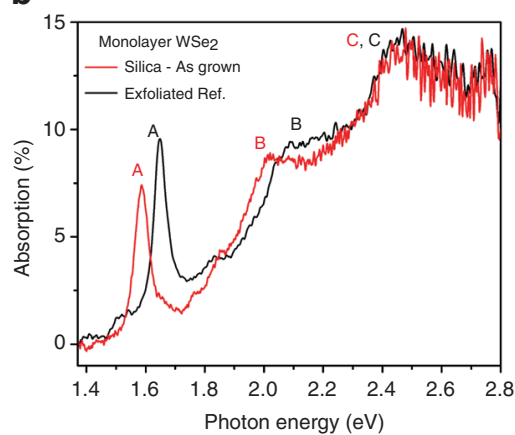

d

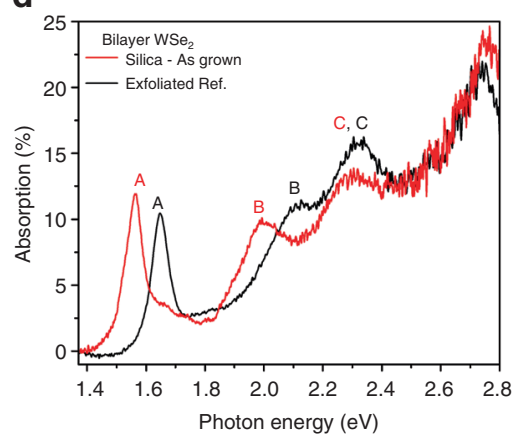

Fig. 4 Photoluminescence of strain-engineered monolayer and bilayer $\mathrm{WSe}_{2}$. a Photoluminescence spectra of as-grown and transferred monolayer WSe as well as exfoliated reference. Inset shows normalized spectra. b Absorption spectra of as-grown and exfoliated monolayer WSe 2 . c Photoluminescence spectra of as-grown and transferred bilayer $\mathrm{WSe}_{2}$ as well as exfoliated reference; inset shows normalized PL spectra of as-grown and transferred bilayer $\mathrm{WSe}_{2}$. d Absorption spectra of as-grown and exfoliated bilayer $\mathrm{WSe}_{2}$

shifted with the presence of tensile strain, and are consistent with the observed shift in the emission. Interestingly, the absorption of the $\mathrm{C}$ exciton peak shows no shift between the as-grown and exfoliated samples, which is consistent with previously reported mechanical bending experiments on exfoliated monolayers, although it should be noted that in these experiments the strain was uniaxial ${ }^{28}$.

More interestingly, it has been previously demonstrated using bending experiments that under the application of a uniaxial strain of $\sim 1.5 \%$, bilayer $\mathrm{WSe}_{2}$ undergoes an indirect-to-direct bandgap transition ${ }^{7}$. This effect is caused by an increase in the energy level of the indirect valley at the $\Sigma$ point as well as a corresponding decrease in the energy level of the direct valley at the $\mathrm{K}$ point ${ }^{7}$. While it is important to note these results are obtained under uniaxial strain, similar trends between uniaxial and biaxial strain have been observed in theoretical studies on other 2D material systems ${ }^{29}$. Figure $4 \mathrm{c}$ depicts the PL spectra of as-grown, exfoliated and transferred bilayer $\mathrm{WSe}_{2}$. In the asgrown strained bilayer, we observe a single sharp PL peak, as oppose to a broader spectrum corresponding to emission from both the indirect and direct bandgaps observed in the unstrained exfoliated and transferred samples. Moreover, the intensity of the $\mathrm{PL}$ is reduced by approximately 50 times upon transfer and resultant release of tensile strain as shown in Fig. 4c. This phenomenon can possibly be attributed to the optical transition from indirect-to-direct in bilayer $\mathrm{WSe}_{2}$ in the presence of tensile strain. This is in good agreement with previous reports on the indirect-to-direct optical transition of $\mathrm{WSe}_{2}$ through bending experiments, although these previous studies utilized uniaxial strain $^{7}$. The normalized PL spectra for bilayer $\mathrm{WSe}_{2}$ grown on fused silica after transfer, as well as unstrained samples grown on sapphire and prepared by exfoliation, are shown in Supplementary Fig. 6 and show a very similar spectral shape. Absorption of $\mathrm{WSe}_{2}$ bilayer samples was also measured and is shown in Fig. $4 \mathrm{~d}$.
To further characterize $\mathrm{WSe}_{2}$ monolayers, we performed low-temperature PL measurements. Numerous studies have experimentally observed that the PL of monolayer $\mathrm{WSe}_{2}$ is quenched at reduced temperatures. This behavior is unusual for a direct bandgap semiconductor and has been attributed to the formation of dark excitons with an energy level $\sim 30 \mathrm{meV}$ below the bright state ${ }^{30,31}$. It has been hypothesized that at low temperatures the bright exciton is allowed to relax to the lower energy level resulting in a reduction of the PL QY. Conversely, both theoretical and experimental studies have suggested that unstrained monolayer $\mathrm{WSe}_{2}$ is an indirect-gap semiconductor, with a small $(<3 \mathrm{kT})$ energy difference between the indirect and direct gaps ${ }^{32}$, ${ }^{33}$. Upon cooling of exfoliated monolayer $\mathrm{WSe}_{2}$ samples, we observe results similar to what has been previously reported as shown in the inset of Fig. 5a, which is a reduction in PL emission at low temperatures. However, for the case of tensile strained monolayer $\mathrm{WSe}_{2}$ as-grown on fused silica we observe the opposite trend (Fig. 5). As the temperature is reduced from 300 to $6 \mathrm{~K}$ the PL intensity increases by over an order of magnitude, resulting in a final PL QY of $14 \%$ as shown in Fig. 5d. The nature of the physical mechanism for the dramatic difference in the temperaturedependent behavior can be directly attributed to band structure modification due to the presence of strain. However, it remains unclear if this is due to an indirect-to-direct bandgap transition or an increase in the energy level of the dark state.

\section{Discussion}

In summary, we have demonstrated controlled and stable strain engineering of $2 \mathrm{D}$ materials by growth, realized through TCE mismatch between the substrate and $2 \mathrm{D}$ material. The demonstrated strain engineering allows for dramatic modulation of the physical properties of $2 \mathrm{D}$ materials. Using $\mathrm{WSe}_{2}$ as a model system, we have achieved a strain induced indirect-to-direct 
a

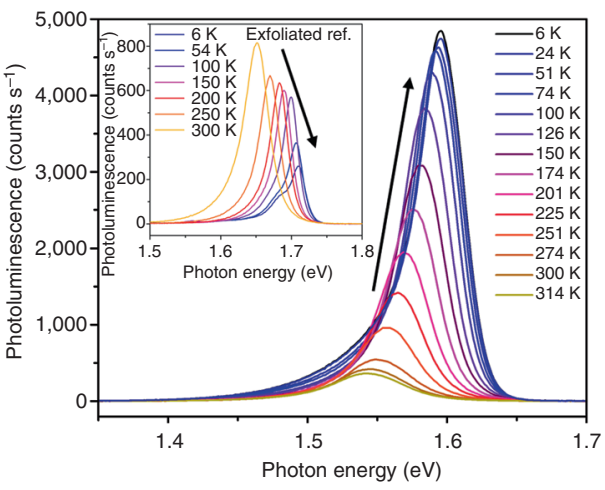

C

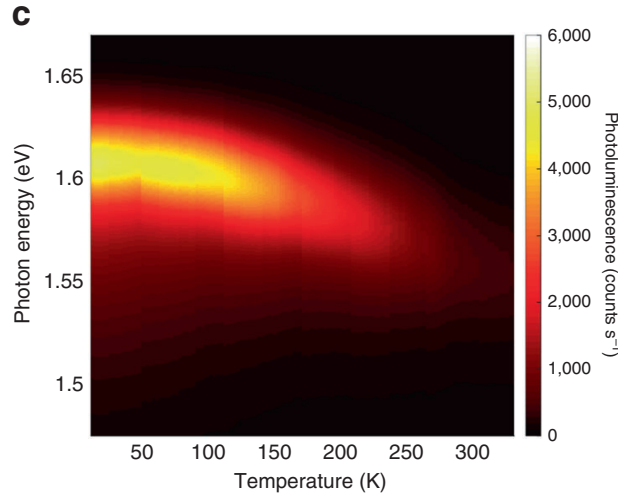

b

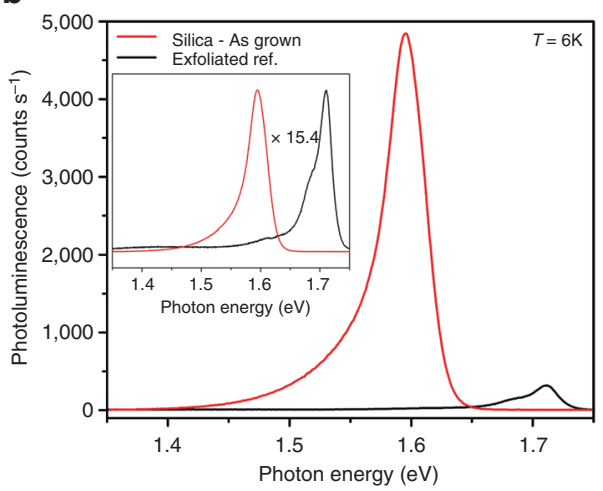

d

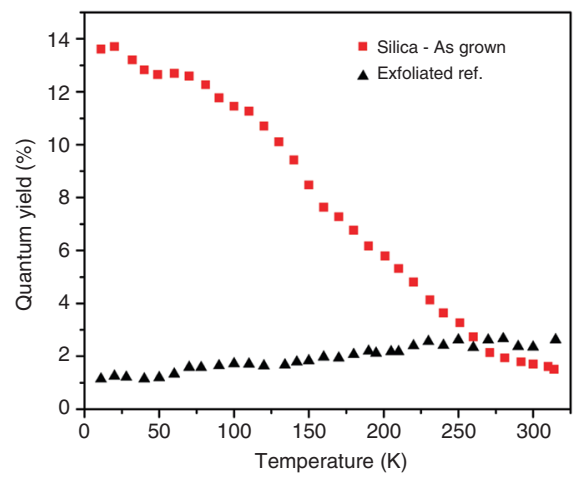

Fig. 5 Low-temperature photoluminescence of strain-engineered monolayer $\mathrm{WSe}_{2}$. a Temperature dependent photoluminescence spectra of as-grown monolayer $\mathrm{WSe}_{2}$ (tensile strained), inset shows the same measurement on an exfoliated monolayer WSe $\mathrm{Sample}$. b Photoluminescence spectra of asgrown (tensile strained) $\mathrm{WSe}_{2}$ and exfoliated $\mathrm{WSe}_{2}$ measured at $6 \mathrm{~K}$, inset shows normalized spectra. c Two dimensional plots of photoluminescence spectra and intensity of as-grown (tensile strained) monolayer WSe $\mathrm{W}_{2}$ d Temperature dependence of the PL QY for as-grown (tensile strained), and exfoliated $\mathrm{WSe}_{2}$ as a function of temperature

optical transition in directly grown $\mathrm{WSe}_{2}$ bilayer, resulting in an amplification of the PL intensity by over fifty times. We have also shown tunable PL emission of $\mathrm{WSe}_{2}$ monolayer, whereas maintaining its high performance. Notably, in the monolayer case under tensile strain, we observed increasing luminescence at reduced temperatures, in stark contrast with what has been reported in the unstrained exfoliated material, suggesting that strain can be utilized to brighten the dark exciton. This practical route for the direct realization of strain engineering in $2 \mathrm{D}$ materials can allow for the characterization of device properties as a function of strain as well as enable optical measurements that are not accessible to samples strained by physical bending or stretching. Our work depicts that biaxial strains of $1 \%$ tensile to $0.2 \%$ compressive can be obtained by using readily achievable differences in the thermal expansion coefficients by choosing an appropriate growth substrate. By expanding upon this method, it may be possible to obtain uniaxial strain via growth on substrates with anisotropic thermal expansion coefficients or obtain significantly higher strain values via growth on piezoelectric substrates $^{34,35}$.

\section{Methods}

Material growth. WSe $e_{2}$ was grown on several substrates with varying thermal expansion coefficients using CVD. Specifically, growth was performed on fused silica (amorphous), aluminum nitride (c-plane), Sapphire (c-plane), or strontium titanate $(<100>)$; all substrates were electronic grade and have a root mean square surface roughness of $<10 \AA$. Substrates were first cleaned by sonication in acetone and isopropyl alcohol for $10 \mathrm{~min}$. The growth was carried out using a two-heatingzone furnace (DaePoong Industry, 50602); a schematic of the growth setup is shown in Supplementary Fig. 7. The cleaned substrates were loaded into the downstream zone, and a ceramic boat containing mixture of $\mathrm{WO}_{3}$ and potassium bromide $(\mathrm{KBr})$ was then placed next to the substrates. $\mathrm{KBr}$ was mixed with $\mathrm{WO}_{3}$ at a ratio of $1: 2$, with the $\mathrm{KBr}$ acting as a promoter for the growth. This is similar to methods described in ref. ${ }^{36}$. However, we found that several salts can be used and the choice of promoter has a significant role in determining the ultimate sample morphology (this is further discussed in the Supplementary Methods). It should be noted that regardless of the promoter used, the strain present in the sample was unaffected as shown in Supplementary Fig. 8. A ceramic boat containing $500 \mathrm{mg}$ Se powder was placed in the center of the upstream zone. After loading, the quartz tube was evacuated and Ar was introduced at $60 \mathrm{sccm}$ resulting in a pressure of 2.6 Torr. The temperature of upstream zone was then ramped to $100^{\circ} \mathrm{C}$ and the downstream furnace was subsequently ramped to a setpoint of $825^{\circ} \mathrm{C}$. The set point of the upstream zone was adjusted such that the residual heat from the downstream zone results in vaporization of Se. Once the temperature of downstream furnace was stabilized, hydrogen was introduced at a flow rate of $40 \mathrm{sccm}$ (total pressure of 3 Torr). This initiates the growth as the presence of hydrogen is required to decompose and vaporize $\mathrm{WO}_{\mathrm{x}}{ }^{37}$. The growth time used was $15 \mathrm{~min}$. Once the growth is complete, hydrogen flow was stopped, and the furnace was opened to rapidly cool the sample. The influence of the $\mathrm{H}_{2} / \mathrm{Ar}$ ratio, growth time and growth pressure is discussed in the Supplementary Methods and are shown in Supplementary Figs 9-11. Optical microscope images of samples grown on various substrates is shown in Supplementary Fig. 12. Specific growth conditions were tuned to optimize the PL QY of the as-grown samples and to either promote growth of bilayer or monolayer domains (discussed in Supplementary Methods). It should be noted that for growths where the substrate temperature was varied the $\mathrm{WO}_{3} / \mathrm{KBr}$ boat was placed in the center of the first heating zone and the Se boat was placed at the edge of the first heating zone. The substrates were placed in the center of the second heating zone. The position of Se boat was adjusted to control the temperature. Transfer of grown $\mathrm{WSe}_{2}$ is done using a $\mathrm{HF}$ based wet transfer technique with PMMA as the transfer medium using the specific procedure described in ref. ${ }^{11}$. The electrical performance of CVD WSe $\mathrm{W}_{2}$ grown on fused silica and transferred to $\mathrm{Si} / \mathrm{SiO}_{2}$ is also compared to that of exfoliated samples, and is shown in Supplementary Fig. 13.

Transmission electron microscopy. WSe $e_{2}$ monolayers were directly grown and transferred on $8 \mathrm{~nm}$ thick $\mathrm{SiO}_{2}$ membranes (Ted Pella) for electron diffraction measurements in order to directly measure the lattice constant of the strained and unstrained samples, respectively. Electron diffraction measurements were 
performed at the National Center for Electron Microscopy at LBNL using a FEI Titan 60-300 microscope operated at $80 \mathrm{kV}$ with parallel beam illumination.

Optical characterization. The PL data presented in this work was obtained using a custom built micro-PL instrument described in detail in ref. ${ }^{1}$. In brief, measurements were performed using the continuous wave $514.5 \mathrm{~nm}$ line of an $\mathrm{Ar}^{+}$laser (Lexel 95) and the laser power was adjusted using neutral density filters. The laser was focused on the sample using an $80 \times$ objective lens $(\mathrm{NA}=0.9)$. The PL signal was measured using the same objective lens, passed through a $550 \mathrm{~nm}$ dielectric longpass filter, dispersed by a $f=340 \mathrm{~mm}$ spectrometer with a $150 \mathrm{~g} \mathrm{~mm}^{-1}$ grating, and detected using by an Si CCD camera (Andor iDus BEX2-DD). Prior to each measurement the CCD background was obtained and subsequently subtracted from the PL acquisition. The sensitivity of the instrument as a function of wavelength (instrument function) was determined through measurement of a virtual Lambertian blackbody source under the objective, created by imaging the illumination from a temperature stabilized lamp (ThorLabs SLS201) on a diffuse reflector (Spectralon). The system efficiency was determined by measuring the response of the excitation laser focused onto a diffuse reflector (Spectralon), with the $550 \mathrm{~nm}$ longpass filter removed. The measured external quantum efficiency was converted to QY using sample absorption at the pump wavelength and the fraction of light, which is able to escape the sample $\left(1 / 4 n^{2}\right.$, where $n$ is the refractive index of the medium). All measurements shown in this work were performed at an incident power of $1.5 \mathrm{~W} \mathrm{~cm}^{-2}$ (corresponding to a laser power of $200 \mathrm{nW}$ ). This laser power is significantly below the onset of biexcitonic recombination in $\mathrm{WSe}_{2}{ }^{38}$. Low temperature measurements were performed using a long working distance $50 \times$ objective lens in a micro-cryostat (Janis) with an infrasil window. The procedure described previously was used to calculate the sample PL efficiency. PL imaging was performed on the samples to determine their uniformity. Measurements were performed using a florescence microscopy setup using a $470 \mathrm{~nm}$ light emitting diode as the excitation source and a CCD detector (Andor Luca). Raman measurements and high-resolution mapping were performed to characterize the strain in the $\mathrm{WSe}_{2}$. Raman spectra were obtained using a WITec Alpha 300RA equipped with a piezo electric scanning stage. The sample was excited using the $532 \mathrm{~nm}$ line of a frequency-doubled Nd:YAG laser as the excitation source and focused on the sample using a $100 \times$ objective.

Data availability. The data that support the findings of this study are available from the corresponding author on request.

Received: 27 February 2017 Accepted: 5 July 2017

Published online: 20 September 2017

\section{References}

1. Amani, M. et al. Near-unity photoluminescence quantum yield in $\mathrm{MoS}_{2}$. Science 350, 1065-1068 (2015).

2. Desai, S. B. et al. $\mathrm{MoS}_{2}$ transistors with 1-nanometer gate lengths. Science 354, 99-101 (2016)

3. Chang, C.-H., Fan, X., Lin, S.-H. \& Kuo, J.-L. Orbital analysis of electronic structure and phonon dispersion in $\mathrm{MoS}_{2}, \mathrm{MoSe}_{2}, \mathrm{WS}_{2}$, and $\mathrm{WSe}_{2}$ monolayers under strain. Phys. Rev. B 88, 195420 (2013).

4. Shi, H., Pan, H., Zhang, Y.-W. \& Yakobson, B. I. Quasiparticle band structures and optical properties of strained monolayer $\mathrm{MoS}_{2}$ and $\mathrm{WS}_{2}$. Phys. Rev. B 87, 155304 (2013)

5. Duerloo, K.-A. N., Li, Y. \& Reed, E. J. Structural phase transitions in two-dimensional Mo- and W- dichalcogenide monolayers. Nat. Commun. 5, 4214 (2014)

6. Zhu, H. et al. Observation of piezoelectricity in free-standing monolayer $\mathrm{MoS}_{2}$. Nat. Nano 10, 151-155 (2015).

7. Desai, S. B. et al. Strain-induced indirect to direct bandgap transition in multilayer WSe $\mathrm{W}_{2}$. Nano Lett. 14, 4592-4597 (2014).

8. Thompson, S. E. et al. 90-nm logic technology featuring strained-silicon. IEEE Trans. Electron Dev. 5, 1790-1797 (2004).

9. Yablonovitch, E. \& Kane, E. O. Band structure engineering of semiconductor lasers for optical communications. J. Lightw. Technol. 6, 1292-1299 (1988).

10. Alamo, J. A. Nanometre-scale electronics with III-V compound semiconductors. Nature 479, 317-323 (2011).

11. Zande, A. M. et al. Grains and grain boundaries in highly crystalline monolayer molybdenum disulphide. Nat. Mat. 12, 554-561 (2013).

12. Yu, J., Li, J., Zhang, W. \& Chang, H. Synthesis of high quality two-dimensional materials via chemical vapor deposition. Chem. Sci. 6, 6705-6716 (2015).

13. Kang, K. et al. High-mobility three-atom-thick semiconducting films with wafer-scale homogeneity. Nature 520, 656-660 (2015).

14. Liu, Z. et al. Strain and structure heterogeneity in $\mathrm{MoS}_{2}$ atomic layers grown by chemical vapour deposition. Nat. Commun. 5, 5246 (2014).

15. Amani, M. et al. Growth-substrate induced performance degradation in chemically synthesized monolayer $\mathrm{MoS}_{2}$ field effect transistors. Appl. Phys. Lett. 104, 203506 (2014).
16. Braun, O. M. \& Kivshar, Y. The Frenkel-Kontorova Model- Concepts, Method, and Applications (Springer, Berlin, 2004).

17. Plechinger, G. et al. Control of biaxial strain in single-layer molybdenite using local thermal expansion of the substrate. 2D Mater. 2, 015006 (2015).

18. Roy, R., Agrawal, D. K. \& McKinstry, H. A. Very low thermal expansion coefficient materials. Annu. Rev. Mater. Sci. 19, 59-81 (1989).

19. Slack, G. A. \& Bartram, S. F. Thermal expansion of some diamondlike crystals J. Appl. Phys. 46, 89-98 (1975).

20. Reeber, R. R. \& Wang, K. Lattice parameters and thermal expansion of important semiconductors and their substrates. Mat. Res. Soc. Symp. 662 T6.35.1-6 (2000)

21. El-Mahalawy, S. H. \& Evans, B. L. The thermal expansion of $2 \mathrm{H}-\mathrm{MoS}_{2}$, $2 \mathrm{H}-\mathrm{MoSe}_{2}$, and $2 \mathrm{H}-\mathrm{WSe}_{2}$ between 20 and $800^{\circ} \mathrm{C}$. J. Appl. Cryst. 9, 403-406 (1976).

22. Ding, Y. \& Xiao, B. Thermal expansion tensors, Grüneisen parameters and photon velocities of bulk $\mathrm{MT}_{2}(\mathrm{M}=\mathrm{W}$ and $\mathrm{Mo} ; \mathrm{T}=\mathrm{S}$ and $\mathrm{Se})$ from first principle calculations. RSC Adv. 5, 18391-18400 (2015).

23. Yim, W. M. \& Paff, R. J. Thermal expansion of AlN, sapphire, and silicon. J. Appl. Phys. 45, 1456-1457 (1974).

24. Ligny, D. \& Richet, P. High temperature heat capacity and thermal expansion of $\mathrm{SrTiO}_{3}$ and $\mathrm{SrZrO}_{3}$ perovskites. Phys. Rev. B 53, 3013-3022 (1996).

25. Late, D. J., Shirodkar, S. N., Waghmare, U. V., Dravid, V. P. \& Rao, C. N. R. Thermal expansion, anharmonicity and temperature dependent Raman spectra of single- and few-layer $\mathrm{MoSe}_{2}$ and $\mathrm{WSe}_{2}$. Chem. Phys. Chem. 15, 1592-1598 (2014).

26. Sahin, H. et al. Anomalous Raman spectra and thickness-dependent electronic properties of $\mathrm{WSe}_{2}$. Phys. Rev. B 87, 165409 (2013).

27. Johari, P. \& Shenoy, V. B. Tuning the electronic properties of semiconducting transition metal dichalcogenides by applying mechanical strains. ACS Nano. 6 , 5449-5456 (2012).

28. Schmidt, R. et al. Reversible uniaxial strain tuning in atomically thin $\mathrm{WSe}_{2} .2 D$ Mat. 3, 021011 (2016).

29. Dong, L., Namburu, R. R., O’Regan, T. P., Dubey, M. \& Dongare, A. M. Theoretical study on strain-induced variation in electronic properties of $\mathrm{MoS}_{2}$. J. Mat. Sci. 49, 6762-6771 (2014).

30. Zhang, X.-X. et al. Experimental evidence for dark excitons in monolayer $\mathrm{WSe}_{2}$. Phys. Rev. B 115, 257403 (2015).

31. Ye, Z. et al. Probing excitonic dark states in single-layer tungsten disulphide. Nature 513, 214-218 (2014).

32. Ramasubramaniam, A. Large excitonic effects in monolayers of molybdenum and tungsten dichalcogenides. Phys. Rev. B 86, 115409 (2012).

33. Zhang, C. et al. Probing critical point energies of transition metal dichalcogenides: surprising indirect gap of single layer WSe $\mathrm{W}_{2}$. Nano Lett. 15, 6494-6500 (2015).

34. Li, J., Bi, W., Ki, W., Huang, X. \& Reddy, S. Nanostructured crystals: Unique hybrid semiconductors exhibiting nearly zero and tunable uniaxial thermal expansion behavior. J. Am. Chem. Soc. 129, 14140-14141 (2007).

35. Chaix-Pluchery, O., Chenevier, B. \& Robles, J. J. Anisotropy of thermal expansion in $\mathrm{YAlO}_{3}$ and $\mathrm{NdGaO}_{3}$. Appl. Phys. Lett. 86, 251911 (2005).

36. $\mathrm{Li}, \mathrm{S}$. et al. Halide-assisted atmospheric pressure growth of large area $\mathrm{WSe}_{2}$ and $\mathrm{WS}_{2}$ monolayer crystals. Appl. Mater. Today 1, 60-66 (2015).

37. Chen, J. et al. Chemical vapor deposition of large-sized hexagonal $\mathrm{WSe}_{2}$ crystals on dielectric substrates. Adv. Matter 27, 6722-6727 (2015).

38. Amani, M. et al. Recombination kinetics and effects of superacid treatment in sulfur- and selenium based transition metal dichalcogenides. Nano Lett. 16 , 2786-2791 (2016).

\section{Acknowledgements}

The authors would like to thank Ian S. Winter for providing them with his elastic constants code that interfaces with VASP. Materials growth, characterization, and modeling were supported by the U.S. Department of Energy, Office of Science, Office of Basic Energy Sciences, Materials Sciences and Engineering Division under contract no. DE-AC02-05CH11231 within the Electronic Materials Program (KC1201). Electron Microscopy was performed at the Molecular Foundry which is supported by the Office of Science, Office of Basic Energy Sciences, of the U.S. Department of Energy under Contract No. DE-AC02-05CH11231.

\section{Author contributions}

G.H.A., M.A. and A.J. conceived the idea for the project and designed the experiments G.H.A. performed the synthesis of WSe $\mathrm{W}_{2}$. G.H.A., M.A. and D.-H.L. carried out the optical characterizations. H.R. and A.M.M. performed electron diffraction measurements. J.P.M. and D.C.C. performed modeling and simulations. G.H.A., M.A., J.P.M., D.C.C., J.W.A., M.D. and A.J. analyzed the data. G.H.A., M.A. and A.J. wrote the manuscript. All authors discussed the results and commented on the manuscript.

\section{Additional information}

Supplementary Information accompanies this paper at doi:10.1038/s41467-017-00516-5. 
Competing interests: The authors declare no competing financial interests.

Reprints and permission information is available online at http://npg.nature.com/ reprintsandpermissions/

Publisher's note: Springer Nature remains neutral with regard to jurisdictional claims in published maps and institutional affiliations.

Open Access This article is licensed under a Creative Commons Attribution 4.0 International License, which permits use, sharing, adaptation, distribution and reproduction in any medium or format, as long as you give appropriate credit to the original author(s) and the source, provide a link to the Creative Commons license, and indicate if changes were made. The images or other third party material in this article are included in the article's Creative Commons license, unless indicated otherwise in a credit line to the material. If material is not included in the article's Creative Commons license and your intended use is not permitted by statutory regulation or exceeds the permitted use, you will need to obtain permission directly from the copyright holder. To view a copy of this license, visit http://creativecommons.org/ licenses/by/4.0/.

(C) The Author(s) 2017 